\title{
HIGHER OREDER CURVATURE GRAVITY IN FINSLER GEOMETRY
}

\author{
N.MEBARKI \\ Laboratoire de Physique Mathematique et Subatomique, Mentouri University, \\ Constantine, 25000, Algeria \\ M.Y.BOUDJADA \\ Space Research Institute, \\ Graz, Austria
}

\begin{abstract}
In Finslerian space-time, a higher order curvature cosmological model of gravity is proposed and a class of cosmological solutions is obtained. Moreover, the viability and stability near critical points are also discussed.
\end{abstract}

\section{Introduction}

During the last few years, considerable studies concerning observable anisotropies of the universe have been investigated. The main motivations are based on the fact that the observed anisotropy of the microwave cosmic radiation is of a dipole type[1]-[3]. This has led to a theoretical interest in the Finsler geometry which is a generalization of the Riemannian spaces[4]-[6]. It is a geometry connecting the metric structure of the space-time to a physical vector field causing an anisotropic structure and an accelerated expanding universe without invoking a dark energy. The goal of this paper is to study the effect of this geometry and related features by considering $f(R)$ theories. Moreover, dynamical behaviors of general solutions are also discussed.

\section{Higher order curvature gravity in Finsler geometry}

Finsler geometry is characterized in the first order of the weak approximation by an order parameter $\mu$ related to the Cartan torsion tensor and describing the anisotropy [6]. Using Palatini formalism in a flat Finsler space with an $f(R)=R^{n}$ action and where its variation is taken with respect to independent osculating metric and affine connection, the generalized conservation law and FRW field equations modulo surface terms lead if $(1+\beta) \delta=3 \mu / 8(n \approx-0.32)$ where $\beta=-3(n-1)^{2} /[2 n(n-2)]$ and $\delta=\mu \beta / 3$ to the following expression of the luminosity distance as a function of the redshift $z$ :

$$
D_{L} \approx \frac{G^{1 / \alpha}}{\alpha H_{0} A C}\left[G_{2} F_{1}\left(C, 1, C+1,-\frac{B}{A} G\right)-{ }_{2} F_{1}\left(C, 1, C+1,-\frac{B}{A}\right)\right] .
$$

Here $B=\mu /(3 H), A=1-B, C=(1+\alpha) / \alpha, G=(1+z)^{\alpha}, \alpha \approx 4.76$ and ${ }_{2} F_{1}(a, b, c, x)$ is the Gauss hypergeometric function. As an illustration, figure 1 displays

\footnotetext{
Work supported by the Algerian Ministry of Education and research.
} 
the scale factor $a / a_{0}$ in terms of the anisotropy parameter $\mu / H_{0}$ for a fixed value of $x=H_{0}\left(t-t_{0}\right)\left(H_{0}, a_{0}\right.$ and $t_{0}$ are the present Hubble constant, scale factor and time respectively). Regarding the metric formulation and if the scale factor has a power law of the form $a=a_{0}\left(t / t_{0}\right)^{\alpha}$, one gets the following non linear system:

$$
\begin{gathered}
n \alpha(3 \alpha-2)+(n-1)[3 \alpha(2 \alpha-1)+2 n(3-2 \alpha)+4 n(n-2)]=0 . \\
12(n-1) \Omega_{1}\left(-\frac{15}{4} \alpha^{2}+\frac{33}{2} \alpha+\Omega_{2}+\Omega_{3}\right)-\Omega_{4}=0 .
\end{gathered}
$$

where $\quad \Omega_{1}=(2 \alpha-1)^{2}, \quad \Omega_{2}=2 n(2 n-2 \alpha-1), \quad \Omega_{3}=3(10-7 \alpha)(6 n-3 n \alpha-4)$ and $\Omega_{4}=n\left(-14 \alpha^{2}+37 \alpha-15\right)$. As a real solution, one has $\alpha \approx 1.03$ and $n \approx 0.55$. Now to study the stability behavior near critical points in the portrait phase space, we take as dynamical variables $x=\dot{R}(n-1) /(R H), v=\mu / H$ and $y=-R(n-1) /\left(6 n H^{2}\right)$ and one

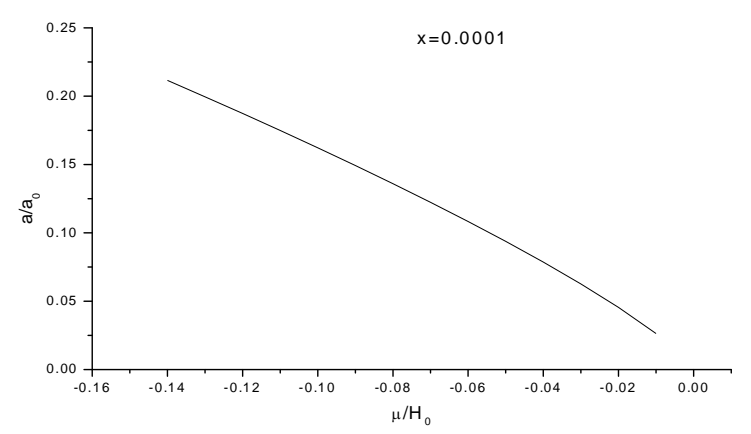

Figure 1. The normalized scale factor as a function of the normalized anisotropy parameter

gets in the vacuum the following autonomous set of differential equations:

$$
\begin{gathered}
x^{\prime}=-\frac{3}{2} v+\left[-\frac{1}{n-1} y-x+\frac{3}{4} v+z+1\right](x+2)+2 y-x^{2} . \\
y^{\prime}=y\left[\frac{3-2 n}{n-1} x-\frac{2}{n-1} y+\frac{3}{2} v+2\right] . \\
v^{\prime}=v\left[1-x+\frac{3}{4} v-\frac{1}{n-1} y\right] .
\end{gathered}
$$

In the $(x, y, v)$ portrait phase space we obtain the following equilibrium points:

1. $A(0,0,0)$ : The stability matrix eigenvalues are $\lambda_{1}=-2, \lambda_{2}=+2$ and $\lambda_{3}=-1$. In this case we have a static open universe. In the $x-y, x-v$ and $y-v$ portrait 
phase space planes, it corresponds to unstable saddle point, stable nodal sink and unstable saddle point respectively.

2. $B(-1,0,0)$ : The stability matrix eigenvalues $\lambda_{1}=2, \lambda_{2}=(4 n-5) /(n-1)$ and

$\lambda_{3}=2$. In this critical point the deceleration and space parameters are $q=+1$ and

$K=0$ (flat space) respectively.

3. $C(2(n-2) /(2 n-1),(4 n-5) /(2 n-1), 0)$ : The deceleration and space parameters are $q=\left(1+2 n-2 n^{2}\right) /[(2 n-1)(n-1)]$ and $K=0$ respectively.

4. $\quad D\left(-2(n-1), 2(n-1)^{2}, 0\right): q=0$ and if $n=-0.78$ or $n=2.78, n\langle-0.78$ or $n\rangle 2.78$ and $-0.78<n<0.78$ we have a flat space, closed and open universe respectively.

5. $\quad E(-11.5,0,-16.47)$ : We have a closed accelerated universe $(q \approx-1$ and $K>0)$.

6. $F(-0.86,0,0.86)$ : We have an accelerated open universe where $q \approx-1.5$ and $K\langle 0$.

\section{Conclusion}

From our qualitative study, we conclude that as a first step towards an understanding of the geometry effect on the cosmology and testing the possibility to get more realistic and viable models, we have found in a flat Finsler geometry with a higher order curvature gravity where $f(R)=R^{n} \quad(n \approx-0.32)$ the following: In the Palatini formalism an explicit exact solution of the Hubble constant in terms of the redshift $z$ and anisotropy parameter $\mu$ and consequently an expression of the distance luminosity is derived. In the metric formulation and by taking a scale factor $a \propto t^{\alpha}$ and contrary to the Riemannian case, the scalar curvature $R$ contains two terms proportional to $1 / t$ and $1 / t^{2}$. Moreover, as a consequence of the generalized conservation law and FWR fields equations, one gets two non linear equations leading to a real solution $\alpha \approx 1.03$ and $n \approx 0.55$ instead of $\alpha$ as a function of $n$ (as it is the case in Riemannian geometry). Concerning the stability behavior, we have obtained more dynamical variables and critical points and the solutions which may be stable in the Riemannian space are not in the Finsler case.

\section{Acknowledgments}

We are very grateful to MESRS for the financial support.

\section{References}

1. E. Ellis and R. Maartens, Class. Quant. Grav. 21, 223 (2004).

2. P. C. Stavrinos, Int. Jour. Theor. Phys. 44, 245 (2005).

3. R. G. Beil, Int. Jour. Theor. Phys. 26, 189 (1987).

4. A.P.Kouretsis, M.Stathakopoulos and P.C.Stavrinos, Phys.Rev.D. 79, 10401 (2009).

5. Z. Chang and X. Li, Phys. Lett. B. 676, 173 (2009).

6. P.C.Stavrinos, A.P.Kourelsis and M.Stathakopoulos, Gen.Rel.Grav.40,1403 (2008). 